\title{
El cometa de Halley y la imagen pública de la astronomía en la prensa diaria española de principios del siglo XX
}

\section{Pedro Ruiz-Castell, Ignacio Suay-Matallana y Juan Marcos Bonet Safont (*)}

$\left(^{*}\right) \quad$ Institut d'Història de la Medicina i de la Ciència «López Piñero», Universitat de València-CSIC. pedro.ruiz-castell@uv.es

SUMARIO: 1.-Introducción. 2.-Los cometas y su controversia. 3.- La prensa ante los temores. 4.- El cometa y las crónicas de sucesos. 5.- Noticias desde Madrid. 6.- Comas i Solà y La Vanguardia. 7.-Consideraciones finales: política y demandas.

RESUMEN: Este texto pretende mostrar algunos aspectos de las representaciones formadas en la opinión pública española acerca de la astronomía y la astrofísica, a partir del análisis de las noticias publicadas en algunas de las cabeceras más destacadas con motivo de la aparición del cometa de Halley en 1910. El interés despertado por la llegada de este cometa estuvo íntimamente ligado a su asociación con diferentes desastres y calamidades, representado en este caso por el equipaje de gases mortíferos que portaba y que desató una inquietud generalizada entre una parte importante de la población. Esta preocupación sirvió para reforzar y legitimar frente a la opinión pública la actividad desarrollada por los científicos, en particular los astrónomos, convertidos en expertos dedicados a tranquilizar a la población mediante datos espectroscópicos, cálculos de mecánica celeste y explicaciones sobre la naturaleza de las masas cometarias, acrecentando el prestigio y estatus social de la astronomía.

PALABRAS CLAVE: historia de la astronomía, cometa de Halley, prensa diaria, España, popularización de la ciencia.

KEY WORDS: history of astronomy, Halley's Comet, newspapers, Spain, popularization of science. 


\section{Introducción $(*)$}

La imagen pública de la ciencia juega un papel fundamental en el desarrollo científico de las naciones. De hecho, la evolución de la ciencia y de los cambios sociales que acontecen en una comunidad determinada está influido por cómo la ciencia se interpreta en ella ${ }^{1}$. En ese sentido, el estudio del papel que juegan los mediadores en los procesos de circulación y comunicación del conocimiento científico resulta especialmente útil e interesante para entender el modo en que la opinión pública demanda y accede a este tipo de conocimiento. Unos procesos de circulación en los que la prensa adquirió a lo largo del siglo XIX una importancia capital ${ }^{2}$.

Los diferentes lenguajes empleados en los trabajos de difusión y popularización científica pueden entenderse como modos especiales y ocasionales de un discurso utilizado por diversas élites para recabar apoyo externo (principalmente moral y financiero) ${ }^{3}$. Así pues, no es de extrañar que los científicos se interesaran, e incluso participaran, de los programas de divulgación científica en la prensa diaria para erigirse como expertos frente a la opinión pública y obtener confianza, poder y legitimación ${ }^{4}$. Gracias a esto la astronomía adquirió un gran atractivo para la opinión pública y ocupó un espacio cada vez más destacado en el imaginario público ${ }^{5}$.

En ese sentido, fenómenos astronómicos como la visita del cometa de Halley en 1910 se convirtieron en oportunidades para reclamar apoyo oficial y social y reconocimiento hacia una disciplina como la astronomía. Algo similar a lo acontecido con motivo de los eclipses totales de sol del

(*) Este trabajo ha contado parcialmente con el apoyo de los proyectos de investigación Física, cultura y política en España (HAR2011-27308), y Entre la ciencia y la ley: Mateu Orfila (17871853), la Toxicología del siglo XIX (HAR2009.12918-C03-03/HIST) Bases para una Historia de la Física en España en el siglo XX (HAR2008-05039), financiados por el Ministerio de Ciencia e Innovación.

1. Pyenson, Lewis; Sheets-Pyenson, Susan. Servants of nature: A history of scientific institutions, enterprises, and sensibilities. New York: W. W. Norton \& Company; 1999.

2. Cantor, Geoffrey, et al. Science in the nineteenth century periodical: Reading the magazine of nature. Cambridge: Cambridge University Press; 2004.

3. Shapin, Steven. Pump and circumstance. Social Studies of Science. 1984; 14: 481-520. Hinton, D. A. Popular science in Britain, 1830-1870. Bath University; 1979.

4. Shapin, Steve; Barnes, Barry. Science, nature and control: Interpreting mechanics institutes. Social Studies of Science. 1977; 7: 31-74. Golinski, Jan. Making natural knowledge. Constructivism and the History of Science. Chicago: The University of Chicago Press; 2005.

5. King, Henry C. Geared to the stars: The evolution of planetariums, orreries and astronomical clocks. Bristol: Adam Hilger; 1978. 
28 de mayo de 1900 y del 30 de agosto de 1905, cuando la gran mayoría de diarios españoles - desde el pro-republicano El Liberal hasta el católico y conservador El Orbe Católico, pasando por otras muchas publicaciones como El Imparcial, el Heraldo de Madrid, la Ilustración Española o Blanco y Negro- recogieron en sus páginas todo aquello relacionado con estos fenómenos astronómicos ${ }^{6}$. Más aún, la presencia en la prensa de estos fenómenos ayudó a difundir unos conocimientos y una imagen favorable de la astronomía entre la población. Un fenómeno que llamó poderosamente la atención de astrónomos y divulgadores extranjeros, quienes lo identificaron con un síntoma de la recuperación social del país ${ }^{7}$.

Así pues, el análisis de las noticias aparecidas en la prensa española de principios del siglo XX nos permite ahondar en las complejas relaciones entre la ciencia y la sociedad española de este período, aproximándonos a aquellos personajes e instituciones que eran consideradas por las élites intelectuales y culturales españolas como las máximas autoridades en relación con el desarrollo de la actividad científica y, en particular, de la astronomía. Del mismo modo, nos facilitan el acceso al conocimiento popular y al imaginario público de la astronomía durante este período, dos factores fundamentales para entender mejor el desarrollo de dicha disciplina en el país durante estas décadas.

Las estrategias de comunicación y divulgación de la astronomía, relacionadas con estos eventos astronómicos, se vieron enmarcadas en un amplio debate público ${ }^{8}$. Un debate que no puede entenderse sin la contribución de la prensa española, caracterizada en este período por un marcado carácter liberal ${ }^{9}$. Con todo, las adhesiones ideológicas de cada publicación habrían de repercutir de manera inevitable en los modelos de divulgación adoptados y en el modo en que fue presentada la ciencia y la actividad de

6. Ruiz-Castell, Pedro. Astronomy and astrophysics in Spain (1850-1914). Newcastle: Cambridge Scholars Publishing; 2008. Ruiz-Castell, Pedro. A «national fiesta»: Total solar eclipses and popularization of astronomy in early twentieth-century Spain. In: Herrán, Néstor et al., eds. Beyond borders: Fresh perspectives in history of science. Newcastle: Cambridge Scholars Publishing; 2008, p. 179-198.

7. Flammarion, Camille. L'éclipse totale de soleil. Bulletin de la Société Astronomique de France. 1900; 14: 289-297. Moreux, Théophile. Autour de l'éclipse: Notes de voyage. Bulletin de la Société Astronomique de France. 1900; 14: 308-324.

8. Nieto-Galán, Agusti. «Not fundamental in a state of full civilization»: The Sociedad Astronómica de Barcelona (1910-1921) and its popularization programme. Annals of Science. 2009; 66: 497-528.

9. Fusi, Juan Pablo; Palafox, Jordi. España: 1808-1996. El desafío de la modernidad. Madrid: Espasa Calpe; 1997. 
los científicos en España ${ }^{10}$. En ese sentido, la línea editorial y las agendas ideológicas de los diarios pueden proporcionar información muy útil para profundizar en estos aspectos e identificar la causa de dicho interés en la difusión y popularización del conocimiento científico en la prensa.

El modo en que la prensa diaria reflejó cuestiones relacionadas con estos eventos astronómicos facilita por tanto el estudio de la difusión y la popularización del conocimiento científico entre un amplio espectro de la sociedad, así como de la imagen pública de la astronomía en este período. De hecho, la amplia repercusión cultural de estos fenómenos astronómi$\cos$-que se puede ver reflejada en diferentes aspectos relacionados con la organización de las observaciones, la convivencia entre astrónomos profesionales y aficionados o los usos de la terminología específica en diferentes contextos- nos permite utilizar estas fuentes para tratar de explorar las diferentes formas en que el conocimiento astronómico se integró como un elemento más de la cultura en la España de principios del siglo XX.

Las siguientes líneas pretenden abordar algunas de estas cuestiones. Lo que aquí se presenta es, en realidad, parte de un proyecto en curso más amplio, en el que colaboran diferentes investigadores españoles, portugueses y griegos, en el marco del grupo STEP con el objeto de realizar un estudio comparado acerca del tratamiento dado por la prensa diaria al paso del cometa de Halley en $1910^{11}$. En el caso español, cuyos primeros resultados se presentan en este artículo, se ha realizado un análisis sistemático de las noticias aparecidas en tres periódicos de gran difusión y representatividad que permiten cubrir un amplio espectro político, social y geográfico: $L a$ Correspondencia de España, El Imparcial y La Vanguardia ${ }^{12}$.

10. González-Silva, Matiana; Herrán, Néstor. Ideology, elitism and social commitment: Alternative images of science in two fin de siècle Barcelona newspapers. Centaurus. 2009; 51: 97-115.

11. El grupo STEP (Science and Technology in the European Periphery) tiene un especial interés en el estudio histórico de la ciencia, la tecnología y la medicina en los contextos tradicionalmente considerados como periféricos. Una de sus líneas de investigación está dedicada a analizar la ciencia en la prensa: http://147.156.155.104/?q=node/483. Sobre el proyecto original dirigido a analizar la imagen de la ciencia y la tecnología proyectada en los diarios de países como Grecia, Portugal, Dinamarca y España: Papanelopoulou, Faidra; Kjærgaard, Peter C. Making the paper: Science and technology in Spanish, Greek and Danish newspapers around 1900. Centaurus, 2009; 51: 89-96. Bonet Safont, Marcos; Suay Matallana, Ignacio; Ruiz-Castell, Pedro. Els públics del cometa Halley (1910): cap a un estudi comparat de l'astronomia en la premsa diària. AUSA. 2012; 25 (169): 537-540.

12. A lo largo del texto se han incorporado también, de forma puntual, noticias procedentes de otras publicaciones para reforzar algunas de las ideas extraídas del análisis sistemático de 
La Correspondencia de España fue el diario español de mayor tirada durante las décadas finales del siglo XIX y principios del siglo XX. Fundado en Madrid en 1859 por el escritor Manuel María Santa Ana (1818-1894), destacó por su marcado carácter conservador ${ }^{13}$. Su gran rival fue el $E l$ Imparcial, fundado en 1867 también en Madrid. Convertido hacia finales del siglo XIX en uno de los periódicos de mayor difusión e influencia de España - gracias en parte a la publicación del suplemento cultural Los Lunes del Imparcial-, mantuvo durante toda su existencia el carácter liberal de su fundador, Eduardo Gasset Artime (1832-1884) ${ }^{14}$. Por último, el diario La Vanguardia fue fundado en Barcelona en 1881 por los hermanos Carles Godó i Pié (1834-1897) y Bartomeu Godó i Pié (1839-1894). Originalmente concebido para apoyar al líder liberal Práxedes Mateo Sagasta (1825-1903), pronto se convirtió en uno de las publicaciones de mayor difusión en Cataluña ${ }^{15}$.

\section{Los cometas y su controversia}

La atención que recibió la visita del cometa Halley en 1910 por parte de la prensa, tanto nacional como internacional, estuvo íntimamente relacionada con el temor que despertó un eventual contacto de la Tierra con

estos tres diarios. Del mismo modo, con la intención de considerar en este estudio una amplia variedad de posicionamientos ideológicos, se consultaron otras publicaciones como Solidaridad Obrera (1907-1919), principal órgano de expresión y propaganda del anarcosindicalismo catalán y Tierra y Libertad (1910-1919), publicación de gran importancia para los grupos anarquistas durante este periodo. Zambrana, Joan. El Movimiento Obrero Catalán en el Periódico Solidaridad Obrera (1907-1919). Cedall: Badalona; 2012. Zambrana, Joan. El anarquismo organizado en los orígenes de la CNT (Tierra y Libertad 1910-1919). Cedall: Badalona; 2012. Sin embargo, en ninguno de estos diarios — considerados esenciales para el estudio de la historia de los movimientos anarquistas en España- hemos encontrado noticia alguna relacionada con el paso del cometa de Halley en 1910.

13. Prieto del Pino, María Dolores. Publicidad, información y opinión del cinematógrafo en la prensa española. Un caso particular: la Correspondencia de España (Madrid, 1896-1921). In: Company, Arnau; Pons, Jordi; Serra, Sebastià, coords. La comunicació audiovisual en la història: Aportacions de la comunicació a la comprensió i construcció de la història del segle XX. Palma de Mallorca: Universitat de les Illes Balears; 2003, p. 895-930.

14. Sánchez Illán, Juan Carlos. Los Gasset y los orígenes del periodismo moderno en España: El Imparcial, 1867-1906. Historia y comunicación social. 1996; 1: 259-276.

15. Martínez, Jesús A. Historia de la Edición en España, 1836-1936. Madrid: Marcial Pons Historia; 2001. 
la cola del cometa. Los astrónomos dudaban acerca de la extensión de la cola del cometa y, por consiguiente, sobre la posibilidad de que nuestro planeta pudiera atravesarla y verse envuelto en ella. Para entender el recelo despertado ante la posibilidad de que la Tierra interaccionara con la cola del cometa de Halley, hay que remontarse al descubrimiento por parte de Daniel Walter Morehouse (1876-1941) en 1908 de un nuevo cometa que, según el análisis espectroscópico realizado del área brillante de su alrededor, portaba diferentes gases, incluyendo algunos altamente tóxicos para las personas, como el cianógeno ${ }^{16}$.

La gran mayoría de astrónomos parecía tener claro que dicha presencia no suponía un peligro ante la visita prevista para 1910 del cometa de Halley, en la que el cometa había de pasar relativamente cerca de la Tierra. Tal y como se apuntaba, la cola del cometa era mucho menos densa que el más perfecto de los vacíos que podían producirse en el laboratorio, por lo que en caso de interacción con ella, la única evidencia observable sería una inofensiva lluvia de meteoros ${ }^{17}$. Sin embargo, los divulgadores científicos habrían de explotar la incertidumbre de un posible paso de la Tierra por la cola del cometa. De hecho, incluso el más conocido y prestigioso divulgador de la astronomía, el francés Camille Flammarion (1842-1925), habría advertido en una noticia publicada en su Anuario Astronómico la posibilidad de un encuentro entre la cola del cometa de Halley y la Tierra el día 18 de mayo, algo que habría podido a ayudar a difundir cierta inquietud entre la opinión pública. Un temor que se encargaría de desmentir poco después en un artículo publicado en Le Petit Journal y que fue también reseñado o incluso reproducido íntegramente en varios diarios españoles ${ }^{18}$.

La presencia de cianógeno en el cometa de Halley abrió un debate acerca de las consecuencias de un hipotético paso de la Tierra por la cola del cometa. Las explicaciones de los diferentes expertos sobre el tema pronto se reflejaron en las publicaciones periódicas nacionales e internacionales,

16. Littmann, Mark; Yeomans, Donald K. Comet Halley: Once in a lifetime. Washington: American Chemical Society; 1985.

17. Campbell, William Wallace. The return of Halley's comet. Publications of the Astronomical Society of the Pacific, 1909; 21: 188-195.

18. Flammarion, Camille. Juicios de Flammarion: Los cometas. La Correspondencia de España. 1 Feb 1910: 2. Flammarion, Camille. [De actualidad] Los cometas. La tarde: diario independiente de noticias y avisos. 5 Feb 1910; 1. Flammarion, Camille. [De actualidad] Los cometas. La Voz de Menorca: Diario Republicano. 10 Feb 1910: 1. 
proporcionando a la astronomía una notoriedad - en parte buscada - que trataría de ser aprovechada por científicos y divulgadores.

La prensa española prestó especial atención a las opiniones vertidas por los científicos franceses y recogió en sus páginas la preocupación por algunas de sus predicciones acerca de los efectos fatales en caso de una hipotética interacción con el cometa ${ }^{19}$. La voz de Flammarion, asegurando lo infundado de los temores de que pereciera el mundo en caso de verse la Tierra envuelta por los gases de la cola del cometa, era reconocida como una de las más autorizadas. Otras como las del director del Observatorio de París, Edouard Benjamin Baillaud (1848-1934), o el director del Observatorio de Meudon, Henri Alexandre Deslandres (1853-1948), confirmaban también que las consecuencias de un posible paso de la Tierra a través de la cola del cometa apenas serían perceptibles para los seres humanos. Sin embargo, algunos científicos reclamaban también, con opiniones contrapuestas a las de estos astrónomos, ser reconocidos como expertos en el tema, aun a riesgo de ser identificados como los causantes de cierta alarma entre la población. Tal y como apuntarían algunos de estos periódicos:

«No cesan los astrónomos, aun sin pretenderlo, de alarmar al público con la afirmación de que del 18 al 19 de Mayo hemos de pasar á través de la cola del cometa de Halley. Por un lado aseguran que nada malo sucederá, y por otra enumeran los peligros que para aquel día nos esperan ${ }^{20}$.

Por ejemplo, el fisiólogo francés Albert Dastré (1844-1917) advirtió de la posibilidad de que si los gases de la cola del cometa de Halley se mezclasen con la atmósfera terrestre, el cianógeno se transformaría en ácido

19. Dicho debate no sólo se reflejó en los tres grandes periódicos que en este texto se analizan, sino también en muchos otros de menor tirada, que reprodujeron y/o copiaron (incluso con errores) las noticias y opiniones procedentes de la vecina Francia. Un Repórter. Notas de un periodista: '¡La fin del mundo!...' El Adelanto: Diario Político de Salamanca. 21 Ene 1910: 2; [¿Un peligro en la atmósfera?] El cometa Halley. El provenir segoviano. 24 Ene 1910: 2; ¿Se acabará el mundo otra vez? La Voz de Menorca: Diario Republicano. 26 Ene 1910: 1-2; [para el 18 de mayo] Más del cometa Halley. El salmantino. 29 Ene 1910: 2-3; El cometa de Halley. El Defensor: Periódico Semanal de Primera Enseñanza. 20 Feb 1910: 4-5.

20. Claravall. Vulgarización científica: ¿Podemos precavernos contra el cianógeno? El Restaurador: diario de propaganda católico-social y de avisos. 7 May 1910: 2. Encontramos otras afirmaciones en la misma línea como la siguiente: «es imposible que la capa gaseosa de la tierra se deje penetrar por la que forma la cola del cometa, siendo, pues, imaginarios los fieros males con que nos amenazaban algunos astrónomos pesimistas». El cometa pavoroso. Gaceta de Mallorca. 25 Feb 1910: 1-2. 
prúsico o cianhídrico, por lo que los seres humanos morirían envenenados instantáneamente. El vicepresidente de la Académie des Sciences, Armand Gauthier (1850-1926), parecía ir más lejos al señalar los efectos tóxicos del cianógeno sin necesidad de transformarse en dicho ácido. Por su parte, el químico francés Albin Haller (1849-1925) advertía de la rápida difusión del cianógeno y cómo, al ser más pesado que el aire, en caso de encontrarse en las alturas de la atmósfera tendería a descender, aunque sin llegar a envenenar a los habitantes de la Tierra puesto que al hallarse mezclado en la atmósfera, bastaría una chispa para producirse una súbita explosión por inflamación del aire. De este modo, las narraciones alarmantes de los diarios sobre las posibles consecuencias del paso de la Tierra por la cola del cometa pudieron valerse de un discurso científico que confería cierta credibilidad a esa inquietud. Tal y como el periodista y escritor Enrique Nieto de Molina (1888-1969) se preguntaba: «[¿]Vencerán las profecías de los astrónomos? ¿Vencerán las de los químicos?» ${ }^{21}$.

Cierta confusión e inquietud se generó con la aparición, en enero de 1910, de un cometa que algunos observadores creyeron que era, dado su brillante núcleo y la extensión de su cola, el cometa de Halley ${ }^{22}$. Igual que sucediera con el cometa de Halley de 1882, el que fuera conocido en un primer momento como cometa de Drake (posteriormente denominado C/1910-A1) fue visto primero en el hemisferio sur —en esta ocasión en Johannesburgo- y pocos días después en Europa, tal y como confirmaron algunos de los observatorios más importantes del momento ${ }^{23}$. Visible incluso a plena luz del día, su aparición en el firmamento desencadenó la publicación de diversas noticias en la prensa relacionadas con estos viajeros celestes, su naturaleza y los fenómenos asociados a estos, algunos de ellos de una inusitada extensión, en los que se abordaba con gran lujo de detalles todas las características de estos objetos celestes ${ }^{24}$.

21. Nieto de Molina, Enrique. El Cometa Halley. Flores y abejas: Revista Festiva Semanal. 13 Feb 1910: 3 (publicado también, sin firma, en El Porvenir de León: Órgano del Partido Republicano. 16 Mar 1910: 1-2).

22. El cometa Halley: Observaciones en Barcelona. El Imparcial. 22 Ene 1910: 2.

23. Blasco, R. Otro cometa a la vista. El Imparcial. 20 Ene 1910: 1. Sobre la confusión del nombre del cometa: Comas i Solà, José. El gran cometa. El defensor de Córdoba. 3 Feb 1910; 1.

24. Vela, A. El cometa de Halley. El Imparcial. 16 May 1910: 1. Vera, V. El cometa nuevo y el cometa de Halley. El Imparcial. 23 Ene 1910: 3 [publicado también en La Voz de Menorca: Diario Republicano. 5 Feb 1910: 1]. 
De hecho, una vez aclarada la confusión inicial, el nuevo cometa apenas se convirtió en un pretexto para plantear cuestiones relacionadas con la esperada - y por algunos temida - visita del cometa de Halley. Tal y como apuntaba el más celebrado astrónomo y divulgador de la astronomía español del momento, Josep Comas i Solà (1868-1937):

«Este cometa [Drake] se ha presentado inopinadamente. Es la antítesis del cometa Halley, que hace 76 años que se le está esperando para darnos nada más que disgustos. Al cometa Drake no le esperaba nadie, y desde luego puede afirmarse que, á pesar de haberse presentado en escena sin preámbulos, va á dejar muy tamañito, en cuanto á aparatosidad, al de Halley» ${ }^{25}$.

Los diarios españoles recogieron también por esas fechas en sus páginas diversas noticias relacionadas con la observación a simple vista desde la Tierra de otros dos cometas: el de Winnecke, descubierto el 31 de octubre de 1909, y el de Daniel, descubierto desde el Observatorio de Halsted (Princeton, EEUU) ${ }^{26}$. Pese a que, tal y como se ha apuntado, la aparición de estos cometas desató la publicación de algunos artículos sobre astronomía cometaria en la prensa, la cobertura específica que se dio a todos ellos fue mucho menor que la dedicada en esas mismas publicaciones al cometa de Halley. Este último era percibido como el más vistoso de todos los cometas y como el que habría de atraer las miradas del público; un verdadero espectáculo digno de contemplar merced a su brillo y a la longitud de su cola $^{27}$. Un cometa periódico cuyo paso por las proximidades de la Tierra podía ser predicho con exactitud por los astrónomos y que, sin embargo, generaba entre una parte de la población cierta alarma y nerviosismo ante las calamidades imprevisibles que parecían acompañarle, tal y como algunos creían que había sucedido en anteriores visitas. La posibilidad de un choque de consecuencias desconocidas entre la Tierra y la cola del

25. Comas i Solà, José. Cometas. La Vanguardia. 23 Ene 1910: 8-9. La cursiva es del original.

26. Blasco, R. El cometa de Johannesburg. Su aparición, su brillo, su forma y su trayectoria. Un año de cometas. El Imparcial. 23 Ene 1910: 3. Aparición de un nuevo cometa. El tiempo: órgano del partido conservador de los distritos de Tortosa y Roquetas. 29 Ene 1910: 2. El cometa Drake. Los debates. 27 Ene 1910: 1. También merece ser mencionada la publicación de la noticia que relataba cómo, en el observatorio de Ginebra, Justin Pidoux había encontrado un nuevo cometa mientras estudiaba los clichés fotográficos realizados durante el seguimiento del cometa Halley. Ginebra. Miscelánea: otro cometa. La Correspondencia de España. 3 Mar 1910: 3.

27. Comas i Solà, José. Notas científicas: un nuevo cometa. La Vanguardia. 29 Abr 1904: 4. 
cometa de Halley terminó de alimentar aún más el interés de unos diarios que cayeron en ocasiones con demasiada facilidad, tanto en España como en el extranjero, en el relato sensacionalista, posiblemente por un afán de ampliar su número de lectores en un contexto de creciente competencia entre publicaciones ${ }^{28}$.

Así pues, de todos los cometas que en 1910 fueron visibles desde la Tierra, el que más atención atrajo por sus posibles efectos, el que realmente se esperaba e interesaba - y a algunos preocupaba-, era el de Halley. La gran mayoría de noticias sobre el temido cometa de Halley fueron publicadas en el mes de mayo de 1910, coincidiendo con el momento en que el cometa pasó más cerca de la Tierra. En dicho mes se concentraron casi la mitad de las noticias publicadas en La Correspondencia de España y un tercio de las aparecidas en El Imparcial y La Vanguardia. A partir de los meses estivales de 1910 el interés informativo fue decayendo y las referencias al cometa Halley se fueron espaciando considerablemente, consistiendo la mayoría en vagas referencias al cometa o a la longitud de su cola. Poco después, en agosto de 1911, las páginas de estos periódicos volverían a prestar atención a la astronomía cometaria con motivo del paso del cometa Kiess ${ }^{29}$.

\section{La prensa ante los temores}

Tal y como se ha indicado, el miedo que despertó en parte de la población el retorno del cometa de Halley en 1910 hizo de la visita de este objeto celeste un tema de actualidad en la opinión pública española. En ese sentido, los tres periódicos muestran la preocupación de una elite educada de clase media por informar e instruir a la población acerca del fenómeno, con el objeto de comprender su naturaleza desde un punto de vista racional, abandonando las supersticiones asociadas a la aparición del mismo a lo largo de la historia de la humanidad. Dicho interés puede entenderse bajo diferentes puntos de vista: desde parte de un ambicioso programa para facilitar una educación liberal a las masas hasta una herramienta de control social. Sea

28. Molina Jiménez, Iván. El paso del cometa Halley por la cultura costarricense de 1910. In: Molina Jiménez, Iván; Palmer, Steven Paul, eds. El paso del cometa: Estado, política social y culturas populares en Costa Rica (1800-1950). San José: EUNED; 1994, p. 231-279.

29. Los viajeros del cielo: El cometa Kiess. La Correspondencia de España. 19 Ago 1911: 1. Comas i Solà, José. Cometa Kiess. La Vanguardia. 1 Ago 1911: 6. 
cual fuere el objetivo final, los científicos y divulgadores participaron de esta retórica, presentando sus tareas de popularización científica como una obligación de gran importancia social, en tanto en cuanto estaban llamadas a incrementar el nivel cultural de la sociedad y revertir de manera positiva en la dignidad y la realización de los individuos ${ }^{30}$.

Tal y como se ha indicado, existía entre la población cierta inquietud con motivo del posible cataclismo que, como consecuencia de un hipotético choque de la Tierra con el cometa de Halley, habría de producirse en la noche del 18 al 19 de mayo de 1910. Con el fin de proporcionar respuesta al desasosiego existente y la expectación generada, los diferentes periódicos publicaron a lo largo de los meses previos al acontecimiento las opiniones de diferentes autores, cuya autoridad sobre la materia quedaba reflejada y/o establecida entre los lectores con la aparición de sus nombres y sus tesis en el periódico.

Entre las afirmaciones de los más eminentes hombres de ciencia recogidas en las páginas de El Imparcial, destacan opiniones tranquilizadoras de ilustres y prestigiosos astrónomos y divulgadores científicos, como Camille Flammarion:

30. Esta preocupación, que se refleja en la gran mayoría de los artículos que han sido analizados para la elaboración de este texto, se enmarcaba en un contexto mucho más amplio, con un cada vez mayor peso de las ideas liberales en distintos países europeos, lo que influiría en el desarrollo de nuevas políticas educativas para extender la instrucción general a todas las clases y estamentos sociales. Esta inquietud se hizo explícita durante las décadas finales del siglo XIX en España, tal y como ponen de manifiesto los trabajos pedagógicos de los químicos José Casares Gil (1866-1961) y José Rodríguez Carracido (1856-1928) o la obra divulgativa de José Echegaray y Eizaguirre (1832-1916). Sánchez Ron, José Manuel, ed. José Echegaray. Madrid: Biblioteca de la ciencia española; 1990. Sánchez Ron, José Manuel. Cincel, martillo y piedra. Madrid: Taurus; 1999, p. 123-151. En cuanto a la astronomía, es bien conocido el interés por la universalización del programa de astronomía popular desarrollado por el astrónomo y divulgador francés Camille Flammarion (1842-1925), así como el destacado papel que jugaron en Gran Bretaña figuras como Richard Anthony Proctor (1837-1888), Agnes Mary Clerke (1842-1907) y Robert Stawell Ball (1840-1913). Sobre los vínculos entre la educación científica, la ciencia popular y la representación de la ciencia en la esfera pública durante la segunda mitad del siglo XIX y principios del siglo XX en Francia y Gran Bretaña: BensaudeVincent, Bernadette; Rasmussen, Anne. La Science populaire dans la presse et l'édition: XIXe et XXe siècles. Paris: CNRS; 1997. Topham, Jonathan R. Scientific publishing and the reading of science in nineteenth-century Britain: a historiographical survey and guide to sources. Studies in History and Philosophy of Science. 2000; 31 A: 559-612. Turner, Frank Miller. Public science in Britain, 1880-1919. Isis. 1980; 71: 589-608. Respecto al caso particular de la divulgación de la astronomía en España, encontramos toda una declaración de intenciones en: Comas i Solà, Josep. Astronomía y ciencia general. Barcelona: F. Granada y Ca; 1907. 
«Flammarion asegura que, los temores de que perezca el mundo el día 18 de Mayo, son infundados. Cree que el acontecimiento no será advertido por el común de las gentes... La observación del fenómeno será interesantísima, y aportará muchos datos al conocimiento de la densidad del núcleo cometario. Acaso produzca el fenómeno perturbaciones eléctricas y magnéticas, auroras boreales, tormentas de un nuevo género, lluvias de estrellas y mágicos resplandores etéreos en las regiones superiores de la atmósfera» ${ }^{31}$.

La astronomía francesa era un modelo a seguir por gran parte de los astrónomos españoles, en especial por los astrónomos aficionados ${ }^{32}$. Muchos de ellos estaban suscritos a las revistas de astronomía y eran miembros de las sociedades astronómicas francesas ${ }^{33}$. No es de extrañar, por consiguiente, que las referencias a las opiniones de los astrónomos de este país encontraran un lugar destacado en la prensa diaria española.

Ya se ha apuntado cómo los diarios españoles mostraron una preocupación constante por el temor infundado que despertaba la visita del cometa, por lo que se esforzaron en mostrar su paso como un fenómeno racional y comprensible. Esta campaña de concienciación e instrucción resultaba especialmente importante a tenor de las declaraciones de algunos científicos acerca de la composición química de la cola del cometa, de la que se habían hecho eco algunos autores populares, tal y como recogieron los periódicos en sus páginas. La noticia de la presencia en la cola del cometa de un gas mortal como el cianógeno hizo correr ríos de tinta en los periódicos, en donde diferentes artículos trataron de mostrar que era infundado el miedo a un eventual paso de la Tierra por la trayectoria del cometa y el contacto con su cola.

El temor que dicha posibilidad despertaba en diferentes sectores de la población mundial fue contrarrestado con argumentos centrados en la baja densidad que poseía el mortífero gas y los efectos irrelevantes que, consecuentemente, habría de tener sobre los humanos y otras formas de

31. Blasco, R. Para el 18 de mayo: Profecías sensacionales. ¿La humanidad en peligro? El Imparcial. 19 Ene 1910: 1.

32. La trayectoria de algunos de los más destacados astrónomos aficionados españoles puede encontrarse en: Oliver, Josep M. Historia de la astronomía amateur en España. Madrid: Sirius; 1997.

33. Nótese cómo los astrónomos aficionados españoles se integraron mayoritariamente en las instituciones concebidas para el desarrollo de la astronomía amateur en Francia, hasta la creación de la Sociedad Astronómica de Barcelona y la Sociedad Astronómica de España y América, fundadas en 1910 y 1911, respectivamente. Ruiz-Castell, n. 6. 
vida en caso de cruzar la Tierra la trayectoria del cometa. Para ello, los diarios recurrieron a la autoridad de prestigiosos astrónomos, tanto locales como extranjeros. Además, la alarma irracional fue ridiculizada en estos diarios de manera constante y continua por parte de científicos, periodistas y corresponsales, tal y como se mostrará más adelante.

Así pues, la preocupación y el interés que despertó el retorno del cometa de Halley pronto convirtió su regreso en un tema de gran actualidad, lo que a su vez atrajo la atención de unos medios de comunicación que se apresuraron a publicar en sus páginas todo tipo de noticias científicas y artículos de divulgación relacionados con el temido visitante. Gran parte de la actividad desarrollada por astrónomos profesionales y amateurs, tanto españoles como extranjeros, quedó plasmada en la prensa, incluyendo aspectos como la organización de expediciones científicas para observar el fenómeno (como la comisión científica internacional enviada a El Teide), las fotografías y resultados obtenidos por los observadores desde diferentes lugares, etc. Dicho seguimiento informativo se vio completado con una extensa crónica social en la que se detallaba toda actividad, evento, incidente o accidente relacionado con la visita del cometa, tanto en España como en el extranjero.

\section{El cometa y las crónicas de sucesos}

En algunos casos, las noticias relacionadas con la expectación que despertaba la visita del cometa de Halley entre el público se confundían con las crónicas de sucesos, que abundaron en los meses previos al encuentro de la Tierra con la cola del cometa. Los periódicos recogieron en sus páginas los diferentes sucesos y las víctimas relacionadas (de un modo más o menos directo) con el paso del cometa. La convicción de la inevitabilidad de un choque entre la Tierra y el cometa, que había de resultar en el fin del mundo, hizo que algunas personas decidieran acabar con su vida antes de la supuesta colisión. Tal fue el caso de varios suicidios cometidos entre febrero y mayo en la ciudad de Valencia ${ }^{34}$. En otros casos, el temor que despertaba la posibilidad de una colisión catastrófica entre la Tierra y el

34. Romero, J. El cometa. El Imparcial. 20 May 1910: 1-2. Una víctima del cometa. El Imparcial. 20 Febr 1910: 2. La expectación del público. El Imparcial. 19 May 1910: 2. 
cometa de Halley angustió a algunas gentes e hizo perder el juicio a más de una persona ${ }^{35}$.

Con todo, las víctimas del cometa no fueron únicamente personajes maníacos, timoratos y pusilánimes. También las hubo entre los curiosos observadores de las clases menos favorecidas que, durante las noches en que el cometa pudo ser observado a simple vista, se encaramaban a lo alto de claraboyas y tejados para contemplar el fenómeno. Algunos de ellos sufrieron desafortunados accidentes y murieron al caer desde las alturas, tal y como se informó en la prensa ${ }^{36}$.

Los diarios también recogieron las supersticiones y creencias de parte del vulgo acerca de las fatalidades que habían de acontecer con la llegada del cometa de Halley. Por ejemplo, la fuerza del temporal que sufrió Galicia a finales del mes de febrero, que desbordó ríos y arroyos - anegando sembrados y casas y arrastrando árboles que derribaban cuanto se oponía a su paso-, alarmó a las gentes del campo, convencidas de que su persistencia se debía a la visita del cometa ${ }^{37}$. Incluso el derrumbe inesperado de una vivienda era atribuido a la influencia del cometa por parte de «los ignorantes campesinos» ${ }^{38}$. El miedo parecía estar instaurado tanto en gentes de campo como de ciudad. Por ejemplo, el corresponsal de El Imparcial en Bilbao recogía con cierto sarcasmo en su crónica del día anterior al paso de la Tierra por la cola del cometa la siguiente información:

«El famoso cometa es el tema obligado de todas las conversaciones. Muchísimas personas ven llegar con verdadero temor el crítico momento, y como prueba de ello, esta mañana pudo verse en las iglesias un extraordinario número de fieles en los comulgatorios. Los sacerdotes, aun procurando ser breves é indulgentes en el tribunal de la penitencia, no pudieron despachar á

35. El cometa de Halley. El Imparcial. 1 May 1910: 3. Noticias por telégrafo (de nuestros corresponsales). El Imparcial. 2 Jun 1910: 3. Trágico fin de un monomaníaco: ¡Que viene el cometa!. La Correspondencia de España. 20 Feb 1910: 1.

36. Romero, n. 34. Noticias por telégrafo (de nuestros corresponsales). El Imparcial. 1 Jun 1910: 3. Suceso trágico: otra víctima del cometa. La Correspondencia de España. 1 May 1910: 3.

37. El temporal. El Imparcial. 24 Feb 1910: 1. Véanse también otras noticias en donde se menciona la relación que en ocasiones se establecía entre el cometa de Halley y la meteorología: Sorpresa. La Correspondencia de España. 3 Abr 1910: 5. Escolástico. De actualidad: El cometa de Halley y la meteorología. La Correspondencia de España. 28 Abr 1910: 1. El cometa y el mal tiempo. La Correspondencia de España. 16 May 1910: 1.

38. Noticias por telégrafo (de nuestros corresponsales): La ignorancia campesina. El Imparcial. 20 Feb 1910: 2. 
todos los que solicitaban confesión, y esta noche estuvieron llenas las iglesias. Mañana habrá una cola caso cometaria ante las sagradas mesas» ${ }^{39}$.

Tal y como reseñan las diferentes noticias y crónicas que fueron publicadas durante aquellos meses, las supersticiones que pretendían combatirse en los artículos publicados en diferentes periódicos, estaban extendidas por todo el mundo, tanto en España como en otros lugares. Los periódicos informaban en mayo de 1910 acerca de cómo la muerte del rey Eduardo VII había sido atribuida por los «ingleses ignorantes» a la influencia del cometa Halley, quienes se mostraban convencidos de que «el cometa es un carro de fuego, enviado por el Ser Supremo para llevar al cielo el alma del rey Eduardo» ${ }^{40}$.

El mismo diario se haría eco pocos días después del espanto que la aparición del cometa generó en diferentes regiones de los Estados Unidos. En particular, se reseñaba el suicidio de una mujer que, aterrorizada por la visita del cometa, se arrojó al mar durante la travesía que realizaba el vapor «Almirante Scheley» desde un puerto del Sur hasta Nueva York, así como las oraciones elevadas al cielo y los extraños ritos celebrados en el campo durante las madrugadas por afroamericanos en el sur de Estados Unidos, con los que pretendían neutralizar la perniciosa influencia del astro errante ${ }^{41}$.

De Salónica llegaban noticias acerca del nerviosismo y el temor que el cometa había despertado en el ex sultán Abdul-Hamid, mientras que desde Italia se informaba de los numerosos casos de locura producidos por el terror, de las enormes cantidades de oxígeno vendidas por boticarios durante aquellos días y de los miles de testamentos redactados por parte de personas convencidas de la parcialidad de la catástrofe y su fatal destino ${ }^{42}$. Por su parte, en La Correspondencia de España se mencionaban los supuestos ataques de una tribu sudafricana a los «usurpadores» blancos,

\footnotetext{
39. El cometa de Halley. El Imparcial. 18 May 1910: 1.

40. La muerte de Eduardo VII. El Imparcial. 11 Mayo 1910: 2.

41. El cometa de Halley, n. 39. Sobre el miedo en otros países: [Ecos] Una noticia de Hungría. La Correspondencia de España. 27 Mar 1910: 1. El temor al cometa: austriacos asustados. ABC. 16 Abr 1910: 7. [La Tierra ha pasado por la cola del cometa: En el extranjero] Otra noticia de Budapest. La Correspondencia de España. 19 May 1910: 2.

42. El cometa de Halley, n. 39.
} 
motivados por las noticias sobre el cometa ${ }^{43}$. Todos estos elementos, junto a los diferentes presagios asociados a la aparición de cometas a lo largo de la historia, fueron enumerados y utilizados por los diarios más liberales como un elemento fundamental de la cruzada que emprendieron contra todas estas supersticiones ${ }^{44}$.

\section{Noticias desde Madrid}

Pese a sus diferencias ideológicas, tanto La Correspondencia de España como El Imparcial abordaron la noticia del retorno del cometa de Halley de forma similar. Ambos diarios publicaron artículos científicos en los que se explicaban, a modo de textos divulgativos, diferentes aspectos relacionados con el cometa y su cola, así como las distintas teorías acerca de su naturaleza y comportamiento postuladas por algunos de los más célebres astrónomos extranjeros. Por ejemplo, en La Correspondencia de España adquirieron un protagonismo especial los astrónomos franceses Camille Flammarion, Théophile Moreux (1867-1954) y Charles Nordmann (18811940), llegándose a publicar traducciones de algunos textos firmados por ellos mismos ${ }^{45}$. Estos autores fueron presentados como los astrónomos y divulgadores más prestigiosos e influyentes del período, convertidos en las páginas de ambas publicaciones en las máximas autoridades sobre la materia.

43. La astronomía y la política: Los Bechuanas se sublevan a causa del cometa. La Correspondencia de España. 22 May 1910: 1.

44. Tal y como afirmarían algunos escritores contemporáneos, «los periódicos liberales habían comenzado una ardorosa campaña en contra de los alarmistas eclesiásticos y del gobierno, benévolo con ellos». Tenreiro, Ramón María. La agonía de Madrid o la cola del cometa: folletín espeluznante. El Cuento Semanal, 1910: Año IV, n. 176.

45. Juicios de Flammarion: Los cometas. La Correspondencia de España. 1 Febr 1910: 2. Pronósticos tranquilizadores: El cometa de Halley. La Correspondencia de España. 12 Ene 1910: 3. Las consecuencias de un encuentro con el planeta. La Correspondencia de España. 31 Ene 1910: 2. El cometa de Halley: Últimas noticias sobre el cometa. La Correspondencia de España. 14 May 1910: 1. Nótese también la presencia de artículos traducidos y firmados por estos autores en otros diarios, como: Moreux, Th. Las consecuencias de un encuentro con el cometa. El Restaurador: diario de propaganda católico-social y de avisos. 3 Febr 1910: 1. Moreux, Th. Consecuencias de un encuentro con el cometa. El Bien Público. 4 Feb 1910: 1. Flammarion, Camille. [Actualidad astronómica] Del cometa. Diario de Tortosa: Órgano del Partido Liberal-Dinástico. 19 Feb 1910: 1. Flammarion, Camille. Otro artículo de Flammarion acerca del cometa. Diario de Córdoba. 25 Abr 1910: 1. Flammarion Camille. El encuentro con el cometa. Heraldo Alavés. 2 May 1910: 1. 
Sin embargo, a medida que se aproximaba la fecha del paso, ambos periódicos comenzaron a recoger más noticias sobre la labor de los astrónomos españoles, ofreciendo una imagen positiva de la profesión y del importante trabajo que realizaban. En este sentido, La Correspondencia de España publicó dos interesantes reportajes sobre los trabajos nocturnos realizados por los astrónomos del Observatorio de Madrid. En uno de ellos se entrevistaba al colaborador del diario y astrónomo auxiliar Enrique Gastardi Peón (1882-1957). Gastardi, que en la década de 1930 llegaría a ser Director General del Instituto Geográfico, Catastral y de Estadística, revelaba en el primero de estos artículos la organización del trabajo y el funcionamiento y la utilidad de telescopios y demás instrumentos científicos existentes en el Observatorio ${ }^{46}$. En el segundo de estos textos destacaba la promoción que se hacía de la institución, incidiendo en los medios disponibles con que contaba para realizar sus investigaciones y algunos de los resultados fotográficos y espectroscópicos obtenidos ${ }^{47}$.

Por otro lado, El Imparcial publicó diversos textos acerca de la naturaleza del cometa. Estas líneas solían estar escritas por Vicente Vera López (1855-1934), quien publicaba artículos divulgativos de manera regular en las páginas de este diario, así como Antonio Vela Herranz (1865-1927), astrónomo del Observatorio Astronómico de Madrid, quien era presentado como una de las principales autoridades en el campo de la astronomía en el país ${ }^{48}$. Estos textos pretendían explicar la naturaleza del fenómeno astronómico y desterrar los miedos que el público pudiera tener en relación con el retorno del cometa de Halley. Sin embargo, cabe destacar el hecho de que para explicar algunos de estos conceptos se empleara un vocabulario técnico, lo que puede ser muestra de las dificultades de algunos de estos primeros periodistas y divulgadores científicos para expresar determinadas ideas y conceptos en términos propios del lenguaje común o bien mostrar

\footnotetext{
46. El cometa de Halley: ¡No pasará nada! De Gobernación al Observatorio. La Correspondencia de España. 12 May 1910: 1.

47. Esperando el paso del cometa. En el Observatorio de Madrid. La Correspondencia de España. 12 May 1910: 1.

48. Vicente Vera fue, probablemente, uno de los primeros periodistas científicos españoles. Doctor en ciencias y catedrático del Instituto de San Isidro de Madrid, fue también químico del Ayuntamiento de Madrid y colaborador de varios periódicos. Ossorio y Bernard, Manuel. Ensayo de un catálogo de periodistas españoles del siglo XIX. Madrid: Imprenta y litografía de J. Palacios; 1904, p. 473.
} 
la familiaridad de una parte importante de los lectores con la terminología especializada que se empleaba.

Durante varias semanas, los diarios recogieron noticias e información relativa a la curiosidad que la visita del cometa despertaba y de las reacciones que a su paso provocaba, tanto en España como en el extranjero. Los diferentes periodistas y articulistas de ambos diarios madrileños incluso animaron a sus lectores a participar de la observación del cometa, recomendando los mejores lugares desde donde contemplar su presencia durante las noches en que debía ser visible. De esta forma, los periódicos adoptaron el papel de guías dedicados a explicar cómo observar el fenómeno y, en cierto modo, invitar a desarrollar un programa de investigación experimental propio de cada observador, en clara alianza con una metodología docente, cada vez más extendida durante aquellos años, que aspiraba a mejorar la educación de la población en diferentes materias científicas y favorecer el ansiado proceso de modernización del país.

El análisis de esta información aparecida en la prensa nos permite explorar los modos en que la actividad científica y los científicos eran presentados y entendidos por parte de los lectores. En este sentido, es particularmente interesante ver que algunas publicaciones como La Correspondencia de España presentaban de forma abierta las controversias y los desacuerdos entre científicos en relación con algunos de los datos obtenidos por parte de los observadores, como la longitud de la cola del cometa y la posible interacción y momento de la Tierra con éste ${ }^{49}$.

\section{Comas i Solà y La Vanguardia}

El estudio de las noticias e informaciones aparecidas en La Vanguardia - con una tirada de unos 50.000 ejemplares en 1910 - merece también una especial atención ${ }^{50}$. En primer lugar, destaca el hecho de que la gran mayoría de artículos publicados por este diario sobre el cometa Halley estaban firmados por el conocido astrónomo y divulgador catalán Josep Comas i Solà. Comas i Solà había cultivado un importante prestigio y reconocimiento como científico y divulgador de la astronomía a partir

\footnotetext{
49. Después del paso. El cometa de Halley. La Correspondencia de España. 27 May 1910: 2.

50. Gómez, Josep Lluís. La gènesi de la premsa de masses a Catalunya, 1902-1923. Barcelona: Pòrtic; 1992.
} 
de la década de 1890, en particular gracias a sus observaciones e investigaciones astronómicas originales, recogidas en publicaciones científicas internacionales como L'astronomie ${ }^{51}$. Algo que le abriría las puertas a unas colaboraciones esporádicas en La Vanguardia que pronto se transformaron en contribuciones asiduas y periódicas ${ }^{52}$.

En cierto sentido, Comas i Solà monopolizó prácticamente toda la información astronómica aparecida en La Vanguardia con motivo de la visita del cometa de Halley en 1910. Con alguna excepción, fue el encargado de redactar prácticamente todos los artículos relacionados con este evento que aparecieron en este periódico ${ }^{53}$. Más aún, si analizamos las noticias sobre nuevas publicaciones o los anuncios e informaciones acerca de conferencias científicas aparecidas en La Vanguardia sobre el cometa, nos encontramos que la autoría de la gran mayoría de estas obras y actividades reseñadas recaía en el propio Comas i Solà. Así pues, si bien es cierto que podemos encontrarnos con algunas menciones a otros científicos y divulgadores, lo cierto es que Comas i Solà es el personaje más citado y que mayor presencia tuvo en las páginas de este diario catalán ${ }^{54}$.

Los artículos de Comas i Solà presentan elementos comunes a los aparecidos en otros diarios. Un buen ejemplo es la crítica a los excesos sensacionalistas de algunas publicaciones en que se hablaba del peligro que entrañaba el paso de la Tierra por la cola del cometa. La intención de Comas i Solà era demostrar que no existía peligro alguno ${ }^{55}$. Ahora bien, sus artículos presentan varias características que no encontramos en los textos de otros autores. En primer lugar, la incorporación de información procedente de los lectores, quienes enviaban con cierta asiduidad al conocido astrónomo y divulgador cartas que en ocasiones eran comentadas por él mismo en la prensa. Una actividad habitual también en algunos de los más prestigiosos divulgadores extranjeros como Flammarion ${ }^{56}$. Esta comunicación fluida con los lectores permitía a Comas i Solà incorporar nuevo

\footnotetext{
51. Roca Rosell, Antoni, coord. Josep Comas i Solà: Astrònom i Divulgador. Barcelona: Ajuntament de Barcelona; 2005.

52. González-Silva; Herrán, n. 10, p. 103.

53. Miret, N. El cometa Halley. La Vanguardia. 13 May 1910: 12.

54. Roig. Ayuntamiento de Barcelona. La Vanguardia. 29 Abr 1910: 4. Notas Regionales. La Vanguardia. 26 Marz 1910: 5. Notas locales. La Vanguardia. 28 Abr 1910: 3.

55. Comas i Solà, José. El cometa Halley. La Vanguardia. 29 Dic 1909: 6. Comas i Solà, José. Cometas. La Vanguardia. 23 Ene 1910: 8.

56. Otro artículo de Flammarion acerca del cometa: Diario de Córdoba. 25 Abr 1910: 1.
} 
material con el que sustentar de manera más convincente sus explicaciones y opiniones, a menudo a partir de los datos procedentes de observaciones realizadas por astrónomos aficionados que le eran comunicadas, así como escribir sobre diferentes temas y aspectos acerca de los cuales sus lectores mostraban un mayor interés ${ }^{57}$.

Además, según se extrae del análisis de noticias publicadas en La Vanguardia, Comas i Solà ignoró la autoridad y reputación de los diferentes astrónomos extranjeros que tan asiduamente eran reconocidos y mencionados en otras publicaciones - como los diarios madrileños-, llegando a criticar duramente a aquellos científicos extranjeros responsables de haber causado alarma entre la población ${ }^{58}$. Es cuanto menos sorprendente, en este sentido, constatar cómo toda la información aparecida en este periódico acerca de los episodios de pánico y reacciones similares causadas por el cometa procedía del extranjero, pero no de España ${ }^{59}$. Aunque quizá más relevante sea el hecho de cómo el ataque de Comas i Solà a algunos de los científicos extranjeros causantes del miedo hacia el cometa fue utilizado para celebrar y apoyar públicamente a los científicos nacionales, empleando una retórica de patriotismo científico:

«Deseo hablar unos momentos de ciencia española (...) Nunca (ni antes, ni ahora) ha tenido España un verdadero plantel de científicos, ni una edad de oro de la ciencia. Y no ha sido seguramente por falta de medios, ni de inteligencia. En cuanto á esta última, está reconocido por todo el mundo que no va á la zaga España de ninguna nación. Basta, para convencerse de ello con fijarse en los tremendos disparates que se publican por los grandes rotativos europeos ${ }^{60}$.

57. Un magnífico ejemplo lo encontramos en la obra El cometa de Halley publicada en 1910 por Comas i Solà. En esta obra, que puede entenderse como una prolongación de sus publicaciones en la prensa, Comas i Solà responde a los comentarios realizados por sus lectores acerca del peligro que pudiera existir en la atracción por parte de la Tierra de partículas cometarias. Véase: Comas i Solà, José. El cometa de Halley. Barcelona: Lopez Robert; 1910, p. 46.

58. Una opinión expresada también, incluso en clave de humor, en diferentes publicaciones como: Un Repórter. «Notas de un periodista: '¡La fin del mundo!...». El Adelanto: Diario Político de Salamanca. 21 Ene 1910: 2. González Pastor; Karikato. Un viaje al cometa Halley, con todas las de la ley (Conclusión). Madrid Cómico. 16 Abr 1910: 9.

59. Los chinos y el cometa. La Vanguardia. 16 Abri 1910: 8. Los temerosos. La Vanguardia. 27 May 1910: 9.

60. Comas i Solà, José. Patriotismo científico. La Vanguardia. 2 Abr 1913: 8. Véase también la afirmación realizada por el mismo Comas i Solà sobre su propia actividad y el estado de 
En este sentido, la falta de referencia alguna a autoridades tan destacadas como Flammarion parece sugerir un intento por parte de Comas i Solà de ser reconocido, al menos por parte del público general, como el astrónomo y divulgador de la astronomía más destacado de este período. Un buen ejemplo de esta estrategia son sus controversias públicas con Eugène Michel Antoniadi (1870-1944) sobre diferentes observaciones planetarias como las de los canales de Marte ${ }^{61}$. De hecho, Comas i Solà encontró en la prensa diaria un importante instrumento de cara a estos debates públicos, cuya razón de ser estuvo íntimamente ligada a su ambición y búsqueda de notoriedad y prestigio en la España de principios del siglo XX.

No en vano, en sus artículos de La Vanguardia, avanzaba algunas de sus ideas más controvertidas con el fin de poder reclamar a posteriori, en caso de mostrarse correctas, la paternidad científica de las mismas. Un buen ejemplo es la hipótesis que formuló en las páginas de este diario en relación con las anomalías observadas en el estudio de la cola del cometa de Halley en $1910^{62}$. De este modo, la publicación de estas suposiciones en periódicos generalistas como La Vanguardia se convirtió en una estrategia común de Comas i Solà con la que atraer la atención de los científicos y del público general, al tiempo que poder reclamar con posterioridad, en caso de ser necesario, el reconocimiento ante la comunidad internacional por su prioridad en el enunciado de dichas ideas.

la astronomía en España: «La Astronomía ha sido en efecto, que yo sepa, la única ciencia que de España que ha repercutido entre las vetustas paredes del Instituto emplazado en la orilla izquierda del Sena. Y no puedo disimular el placer con que oía, allí dentro, asegurar que, hasta ahora, no son conocidas observaciones más interesantes del cometa de Halley que las efectuadas en el Observatorio Fabra». Comas i Solà, José. Impresiones científicas. La Vanguardia. 15 Ago 1910: 6.

61. Ruiz-Castell, Pedro. Priority claims and public disputes in astronomy: E.M. Antoniadi, J. Comas i Solà and the search for authority and social prestige in the early twentieth century. British Journal for the History of Science. 2011; 44: 509-531.

62. Comas i Solà, José. Algo más sobre el gran cometa. La Vanguardia. 13 Feb 1910: 8-9. Nótese que este tipo de actuación no se restringía únicamente a los diarios y se detecta también en otro tipo de publicaciones divulgativas. Por ejemplo, en su monografía El cometa de Halley, tras indicar como pudo observar y fotografiar desde el observatorio Fabra el nuevo cometa aparecido en enero de 1909, Comas i Solà avanza una nueva teoría acerca del brillo de la cola de dicho cometa. Comas i Solà, n. 57, p. 20. 


\section{Consideraciones finales: política y demandas}

El paso del cometa despertó un enorme interés que quedó reflejado en el uso que se hizo del fenómeno astronómico para abordar cuestiones políticas y reivindicar actitudes e iniciativas tanto personales como referentes a la disciplina. Desde principios de 1910, la astronomía cometaria se convirtió en un tema de conversación recurrente en la sociedad española. El público profano pudo aproximarse e informarse, gracias a diferentes publicaciones y medios de comunicación como la prensa diaria, a diferentes cuestiones relacionadas con la visita de cometas como el de Halley, familiarizándose tanto con la jerga astronómica como con diversas cuestiones asociadas a este objeto celeste. La prueba de esta familiaridad la encontramos en las crónicas políticas y de sociedad realizadas por diferentes autores en estos mismos periódicos, tal y como ponen de manifiesto las informaciones en donde se hacía referencia al cometa de Halley para describir las vicisitudes de la actividad política española de este período ${ }^{63}$.

El regreso de este cometa en 1910 se convirtió en un tema de gran presencia pública y actualidad. En este sentido, podemos concluir que la gran mayoría de la población conocía las calamidades a las que el cometa se había asociado a lo largo de la historia de la humanidad, anunciadas de nuevo por algunos catastrofistas. No es de extrañar, por tanto, que el cometa se convirtiera en un recurso de lo más útil para tratar otras cuestiones más allá de la astronomía. Un buen ejemplo son las reflexiones y conversaciones, algunas de ellas reflejadas en la prensa, en las que dichas asociaciones catastróficas se revelaban como una afilada arma dialéctica con la que representar la inestable situación política del país, incluyendo el fracaso y el cese de diferentes políticos y gobiernos. Sin duda alguna, los lectores de estos comentarios conocían a la perfección los diferentes aspectos con los que la visita del cometa se había identificado tradicionalmente.

El caso de La Correspondencia de España es particularmente interesante por el uso repetido que se realizó en este diario de referencias y metáforas relacionadas con el cometa de Halley para abordar todo tipo de temas, en especial los relacionados con la situación política del país, muchos de ellos con un marcado carácter irónico ${ }^{64}$. Se trata de una buena muestra no sólo

\footnotetext{
63. Rovira. El conde de Romanones. La Vanguardia. 1 Abr 1910: 7.

64. La Tierra ha pasado por la cola del cometa. En Provincias. La Correspondencia de España. 19 May 1910: 1. El cometa. La Correspondencia de España. 20 May 1910: 3. La redacción de
} 
de la presencia en el debate público de diferentes aspectos relacionados con la visita del cometa y las calamidades que algunos individuos asociaban a su presencia en los cielos, sino también de la familiaridad del público general con gran parte de la terminología científica asociada a las descripciones astronómicas de este fenómeno celeste. Más aún, en una cabecera de carácter conservador como La Correspondencia de España, destacan algunos intentos por reconciliar la ciencia con la religión, tal y como se extrae de la noticia relativa a la falsedad de la leyenda acerca de la excomunión del cometa de Halley por el papa Calixto III $^{65}$.

La familiaridad de la sociedad española con diferentes aspectos astronómicos se puso también de manifiesto en las representaciones con que contaron en diferentes celebraciones populares como el carnaval, donde ese mismo año desfiló por Madrid una carroza dedicada al cometa de Halley, formada por una media luna entre nubes frente a la que se situaban varias estrellas representadas por muchachas y tras la cual se representaba a varios astrónomos contemplando las constelaciones con grandes catalejos ${ }^{66}$. Del mismo modo, la información obtenida en La Correspondencia de España destaca el gran esfuerzo realizado en la Exposición Nacional de Valencia - continuadora de la Exposición Regional de 1909- para popularizar diferentes aspectos relacionados con la naturaleza del cometa, mediante la instalación de un observatorio provisional y la proyección de las distintas fases de acercamiento del cometa, animando a los espectadores a rellenar boletines con los datos recogidos. Todo ello para poder comunicar «a las gentes de 1985 de qué modo y manera supimos en 1910 combatir la ignorancia y la superstición» ${ }^{67}$. Esta percepción y representación de la astronomía y de los astrónomos, así como de las actividades que realizaban y que fueron detalladas en los periódicos, merecen una mención especial, en tanto en cuanto se erigen en elementos cruciales para reconstruir la

textos para responder a las repetidas preguntas del público general fue práctica habitual en otras formas de popularización, como observamos en: Ubach, José. El cometa de Halley: su paso cerca de la tierra. Barcelona: Gustavo Gili; 1910, p. 5.

65. Del Cacho, José. El cometa de Halley: La leyenda de su excomunión. La Correspondencia de España. 14 May 1910: 1. Estos intentos eran compartidos por otras cabeceras también de corte conservador. Un buen ejemplo lo encontramos en la siguiente cita: «Con aumentar la densidad del cianógeno ¿qué sería de todos los pecadores, impíos é indiferentes? ¡Oh poder de Dios! ¡Oh misericordia divina!». El cometa. La Defensa. 3 May 1910: 2.

66. El carnaval. El Imparcial. 7 Feb 1910: 1.

67. Desde Valencia: El cometa en la Exposición. La Correspondencia de España. 16 May 1910: 3. 
imagen pública de la astronomía y de los astrónomos durante las primeras décadas del siglo XX.

La visita del cometa sirvió también para reclamar un mayor reconocimiento y estatus social para la actividad científica en el país. Gracias a la ciencia y a los científicos, el pueblo ya no tendría motivo para temores, recelos o supersticiones ${ }^{68}$. Pero además, los diarios se convirtieron en espacios donde demandar una mayor inversión económica y un mayor apoyo para el desarrollo de una actividad científica que parecía desarrollarse en España con importantes deficiencias. Un buen ejemplo es la denuncia relacionada con la falta de instrumentos adecuados para realizar investigaciones por parte del catedrático de astronomía de la Universidad de Zaragoza, Gabriel Galán y Ruiz (1869-1938) ${ }^{69}$. En el mismo sentido se encuentran las declaraciones de Comas i Solà sobre la falta de recursos y financiación necesaria para disponer de instrumentos científicos óptimos con los que mejorar sus investigaciones ${ }^{70}$. Todas estas demandas se habían producido con anterioridad con motivo de los eclipses de Sol de 1900 y 1905, momento en que otros diarios como El Liberal actuaron como caja de resonancia de la opinión de un importante número de miembros de la elite social y cultural del país que reclamaban a los gobernantes un apoyo unívoco y la promoción de la ciencia y de la educación científica con el fin de desarrollar la infraestructura económica sólida necesaria para una economía moderna llamada a regenerar el país ${ }^{71}$.

La opinión pública española estuvo ampliamente informada durante la primera década del siglo XX acerca de diferentes cuestiones científicas, que a menudo se convirtieron en tema de conversación en reuniones de todo tipo. El papel de la prensa fue fundamental para la creación y consolidación de los imaginarios científicos y tecnológicos en la sociedad española de prin-

68. Esperando el paso del cometa: explicación de una teoría. La Correspondencia de España. 17 May 1910: 1. Del Cacho, José. El cometa: En Sirio y en Serio. La Correspondencia de España. 18 May 1910: 1. O como afirmaría otro articulista: «iBendita sea la ciencia, que trae tranquilidad á nuestro perturbado ánimo!». Noria, F. Casos y Cosas. Diario de Tortosa: Órgano del Partido Liberal-Dinástico. 2 Abr 1910: 1.

69. Romero, n. 11. Las deficiencias del material científico fueron denunciadas también por otros diarios. Pasatiempos. ABC. 4 Jul 1910: 13.

70. Comas i Solà, José. Un poco de sismología práctica. La Vanguardia. 6 Ene 1911: 6. Véase también la queja de Comas i Solà por no contar con una oficina de calculistas de élite «cuyos resultados serian un título de gloria para sus colaboradores y un titulo de honor para España». Comas i Solà, José. Cometas. La Vanguardia. 23 Ene 1910: 8-9.

71. Ruiz-Castell, n. 6. 
cipios del siglo XX. El completo tratamiento informativo realizado sobre el cometa de Halley en 1910 no sólo implicó la divulgación de conocimientos astronómicos, sino que también fue aprovechado como una herramienta de negociación y legitimación por los astrónomos, quienes perseguían tanto la mejora de sus medios materiales de investigación como la consolidación de su especialidad y el reconocimiento público de su autoridad ${ }^{72}$. En ese sentido, la unión de los intereses personales y profesionales de los científicos junto con los de los medios de comunicación influyó de forma crucial en el desarrollo del debate público y en la toma de decisiones de diferentes gobiernos que, impregnados del espíritu regeneracionista, favorecieron un cambio gradual, en lo social y en lo cultural, del que la actividad científica participó activamente durante el primer tercio del siglo XX.

\section{Agradecimientos}

Este trabajo forma parte de un proyecto de investigación más amplio en el que participan también sendos grupos de investigadores portugueses y griegos. Nuestra gratitud a todos ellos, en especial a Ana Simões y Faidra Papanelopoulou, por el continuo intercambio de ideas. Además, parte de este artículo fue presentado en diciembre de 2011 en el simposio «Historia de la física en España en el siglo XX: balance y perspectivas» celebrado en Barcelona. Queremos agradecer a todos aquellos que con sus comentarios ayudaron a mejorar aquella primera versión de este artículo, así como a José Ramón Bertomeu Sánchez por el apoyo prestado para poder realizar esta investigación.

72. La fiesta de la ciencia. El Imparcial. 29 May 1910: 1. 


\section{ARTICLES}

Halley's Comet and the public image of astronomy in the Spanish daily press of the early 20th century

Pedro Ruiz-Castell, Ignacio Suay-Matallana and Juan Marcos Bonet Safont

1.- Introduction. 2.-Comets and their controversy. 3.- The press in the face of fears. 4. - The comet and press accident/crime reports. 5. - News from Madrid. 6.- Comas i Solà and La Vanguardia. 7.-Final considerations: policies and demands.

ABSTRACT: The aim of this paper was to show some aspects of the representations formed in Spanish public opinion on astronomy and astrophysics, based on an 
analysis of the news published under some of the most prominent headlines on the appearance of Halley's Comet in 1910. The interest aroused by the arrival of this comet was closely bound to its association with different disasters and calamities, represented in this case by the load of lethal gas it carried and that generated widespread unease among a large part of the population. This concern served to reinforce and legitimize in the eyes of public opinion the activity developed by scientists and especially astronomers, who became experts devoted to calming the population with spectroscopic data, calculations of celestial mechanics, and explanations of the nature of comet masses, thereby increasing the prestige and social status of astronomy. 